九州大学学術情報リポジトリ

Kyushu University Institutional Repository

\title{
Applicability of interactive evolutionary computation to mental health measurement
}

Takagi, Hideyuki

Faculty of Design, Kyushu University : Professor

Takahashi, Tomohiro

School of Medicine, Kurume University

Aoki, Ken

Faculty of Human and Social Environment, Hiroshima International University

http://hdl. handle. net/2324/1670075

出版情報: Conference Proceedings - IEEE International Conference on Systems, Man and Cybernetics. 6, pp.5714-5718, 2004-10-10. IEEE

バージョン :

権利関係 : 


\title{
Applicability of Interactive Evolutionary Computation to Mental Health Measurement*
}

\author{
Hideyuki TAKAGI \\ Faculty of Design \\ Kyushu University \\ Fukuoka, Fukuoka, 815-8540 Japan \\ takagi@design.kyushu-u.ac.jp
}

\author{
Tomohiro TAKAHASHI \\ School of Medicine \\ Kurume University \\ Kurume, Fukuoka, 830-0011 Japan \\ tomo-takahashi@k6.dion.ne.jp
}

\author{
Ken AOKI \\ Faculty of Human and Social Environment \\ Hiroshima International University \\ Kurose-cho, Kamo-Gun, Hiroshima 724-0695 Japan \\ k-aoki@he.hirokoku-u.ac.jp
}

\begin{abstract}
We show experimentally the applicability of interactive evolutionary computation (IEC) to a new application field, mental health measurement. We had 3 schizophrenics and 5 mentally healthy students design happy and sad impression computer graphics (CG) lighting images using IEC and asked other 33 students to evaluate the CG images using Scheffe's method of paired comparison. Statistical tests of the evaluations showed that the range of emotional impressions perceived by the three schizophrenics between happy-sad was significantly narrower than that which was perceived by the mentally healthy students $(p<0.01)$. This experimental result implies that IEC has potential to contribute to psychiatry, and it also showed the possibility to expand IEC applicability beyond conventional system optimization and to a new area, mental health measurement.
\end{abstract}

Keywords: interactive evolutionary computation, schizophrenia, mental health measurement, CG lighting.

\section{Introduction}

Interactive Evolutionary Computation (IEC) is a method for optimizing target systems based on human evaluation criteria (see Figure 1). IEC optimizes a target system based on the IEC user's psychological scale, while conventional optimization methods optimize target systems based on cost functions or fitness functions. IEC therefore provides us with a framework for system optimization even in such cases where the optimization tasks are based on personal preference or evaluation, which is difficult to describe mathematically.

\footnotetext{
${ }^{*}$ 0-7803-8566-7/04/\$20.00 C 2004 IEEE.
}

IEC research has come to be used in a wide variety of fields over the past decade, such as: graphic arts and animation; 3-D CG lighting; music; editorial design; industrial design; facial image generation; speech processing and synthesis; hearing aid fitting; virtual reality; media database retrieval; data mining; image processing; control and robotics; MEMS design; food industry; geophysical simulation; education; entertainment; social systems and others [4].

In this paper, we propose a new application for IEC in the measuring of mental health by observing the output of an optimized system. The objective of conventional IEC approaches was to design or optimize target systems. On the other hand, the objective of the proposed method is to characterize an IEC user's mental health by observing the IEC system's output after it has been optimized according to the user's psychological evaluation scale. In the next section, we show how to realize this mental health measurement and in sections 3 and 4, we conduct an experiment to evaluate the applicability of this mental health measurement to psychiatry.

\section{Mental Health Measurement with IEC}

Let us shift our view point from system optimization, which has been the main objective of IEC (Figure 1(a)) until now, to the IEC user whose psychological scale is used to optimize target systems (Figure 1(b)). The IEC user's psychological evaluation scale is reflected in his or her subjective evaluation characteristics, and these in turn, are reflected in the systems designed with IEC. We may therefore observe the psychological scale or the mental health state of the user indirectly by observing the system outputs. This constitutes a new direction for IEC research. 


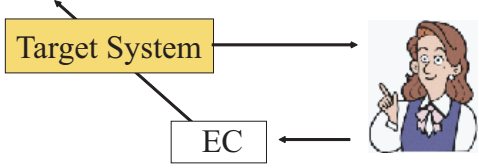

(a)

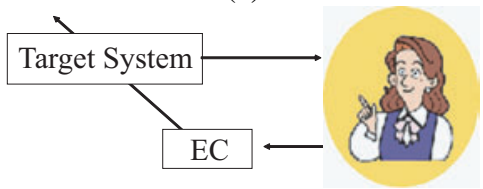

(b)

Figure 1: IEC framework. (a) The objective of conventional IEC is to optimize a given target system based on human subjective evaluation. (b) The proposed usage of IEC is to observe the mental health of an IEC user indirectly by observing the system's output (which reflects the IEC user's psychological evaluation scale).

Although there are many measurable aspects of mental health, for our first trial, in this paper IEC is used to measure the dynamic range of the IEC user's perception of emotional expression. This proposed approach has the potential to provide useful information to psychiatric diagnosticians.

It is said that, through their experience, psychiatrists and psychotherapists feel that schizophrenics have a problem with emotional expression, but they have not been able to use their observation to aid in diagnosis because there is no quantitative way to measure mental health or emotional expression capability.

It is our hypothesis that the dynamic range of emotional expressions perceived by schizophrenics for the distance between happy and sad, happy-sad, is, for example, narrower than that perceived by healthy persons. In our paper, we experimentally confirm this hypothesis using our proposed approach.

This approach consists of two parts, first we give two emotional motifs to IEC users and let them design the motif using an IEC-based design system, and secondly we conduct a subjective test for the obtained designs, construct a psychological scale, and evaluate the difference between these designs statistically.

By conducting this experiment for paired emotion motifs, we can, for example, measure the emotional expression range of a certain subject and compare it with that of other subjects. Once we can obtain such measured data, they may be useful for psychological counseling or psychiatry. Figure 2 illlustrates this concept.

In our experiment, we had IEC users design happy and sad impressions using a 3-D CG lighting design support system $[1,2]$. If the designed $C G$ images were to depend on the $C G$ skills of subjects, we could not estimate the dynamic range of emotional expression by comparing these CG images. This is why we use an IEC system, which does not require CG

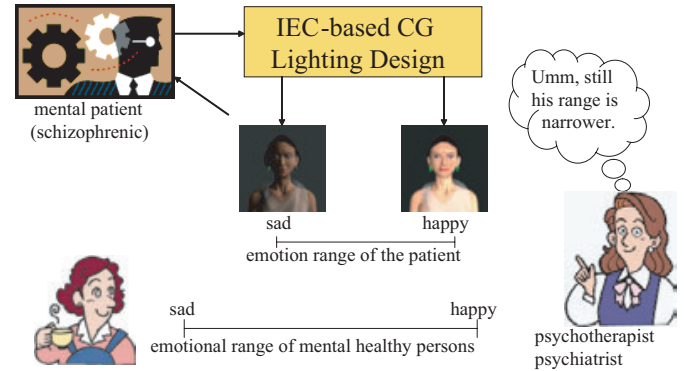

Figure 2: Mental health measurement using an IEC-based design support system. A subject designs impressions, such as happy and sad, using an IEC-based design support system, and mental experts compare his or her impression range of happy-sad with that of mentally healthy subjects.

modeling familiarity or any other creative skill, was needed.

One of the possible implementations we chose to explore for this paper involves three steps:

1. have several subjects create computer graphics (CG) that match the given design motif, such as happy or sad (section 3.2),

2. have several other normal subjects evaluate the difference in emotional expression between the CG-created images though subjective tests (section 3.3), @

3. estimate the psychological scale of perceived emotional expression based on the analysis of variance of the data in the 2 .

\section{Measuring the Ability of Schizophrenics to Perceive Emo- tional Expression}

\subsection{Description of IEC Users for Perceived Emotional Expression Measurement}

Subjects consisted of three schizophrenics and five mentally healthy students, none of whom are colorblind. The three schizophrenics, PM, PT, and PK, understood what was expected, agreed to be our experimental subjects, and signed agreements by themselves several days after our explanation. PM, PT, and PK are: a 27 year-old outpatient, a 41 year-old inpatient of 11 years and 5 months, and a 48 year-old inpatient of 4 years and 6 months, respectively. There had been no changes in their symptoms or drug regimes for a minimum of half a year prior to the experiment. Their symptoms and daily-life functions were measured using PANSS [3] and LASMI, respectively. Five mentally healthy subjects, NS, NN, NK, NY, and NH, were undergraduate and graduate school students. 


\subsection{Measurement of Perceived Emotional Ex- pression with IEC-based Design}

To measure emotional expression we adopted a 3-D CG lighting design support system $[1,2]$ to create $\mathrm{CG}$ images suitable for the experimental design.

This system is an IEC system that interactively optimizes the parameters of three lights using genetic algorithms (GA). These parameters are: coordinates of the lights in a 3-D space, lighting strength, color, and types of light sources. Subjects were requested to evaluate how closely nine displayed differently lit CG images of a 3-D woman were to the given design motif using a scale with five levels. These subjective evaluation values were fed back in to the GA and the procedure was repeated until satisfactory lighting design was obtained.

Figure 3 shows a schematic of the IEC design experiment that was used to measure the emotional expression of the user. Each user was presented with nine individuals generated by the CG lighting design IEC program. They were instructed to identify the most happy individual with a grade 5 and the least happy individual with a grade 1 when happy design motif is given. Same instruction is given when sad design motif is given, too. They were then instructed to rate the remaining seven designs from 1 to 5 . When all nine individuals had been rated, the GA generated nine new individuals for evaluation, and the process repeated. After 10 to 11 generations, the examiners stopped the evolution and recorded the most happy (or the most sad) individuals from the final generation.

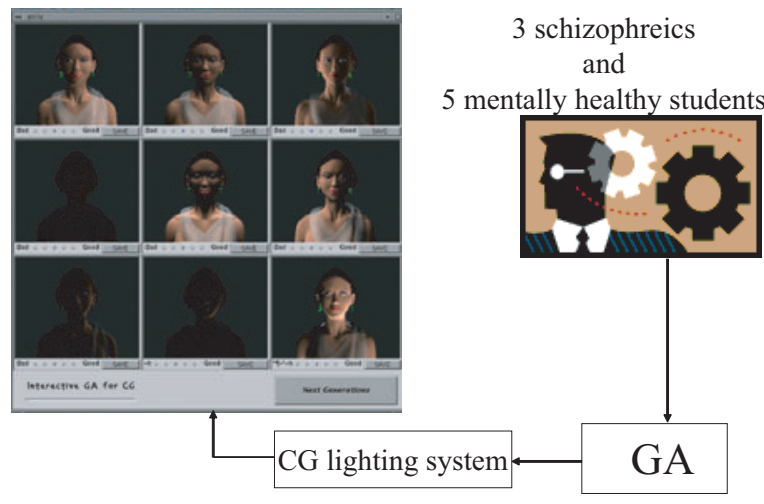

Figure 3: The graphical user interface of the IEC-based CG lighting design support system. Three schizophrenics and five mentally healthy subjects compared nine lighting designs and evaluated them according to a scale with five grades. A genetic algorithm optimized a CG lighting system based on their evaluations.

\subsection{Subjective Test and Construction of Psy- chological Scale}

The best lighting designs for each subject were collected from the first experiment. These happy and sad designs were evaluated by a second set of subjects using Scheffé's method of paired comparison, and a psychological scale was constructed. Statistical significance among the best lighting designs was judged by calculating a psychological yardstick. Through this subjective evaluation and statistical test, we can say, for example, that the lighting of the happy impression designed by the subject A looks happier than that designed by the subject B significantly.

Thirty-three other evaluators, 25 males and 8 females of combined average age of 21.9 years old, evaluated the 28 pairs $\left(={ }_{8} C_{2}\right)$ of happy $\mathrm{CG}$ lighting designs obtained from the best 8 made by the subjects of section 3.1. Using a five-level rating scale, the scores were analyzed using the Scheffé's method of paired comparison with Nakaya's variation (Figure 4). The same evaluation was conducted for the sad CG lighting designs. These psychological experiments were conducted in accordance with the experimental guidelines and under the direction of examiners in order to keep consistent experimental conditions.


28 happy pairs of 8 subjects 28 sad pairs of 8 subjects

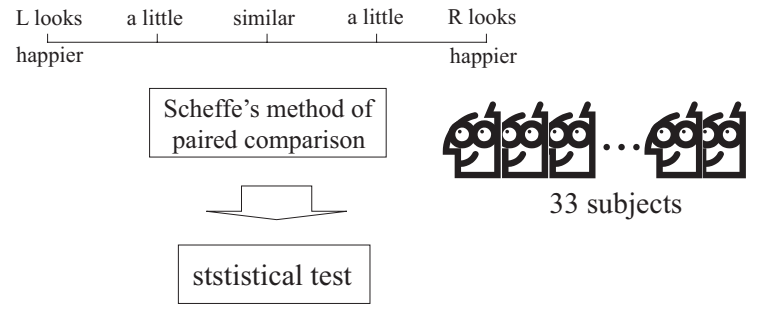

Figure 4: Thirty-three subjects used a five-level rating scale to compare 28 pairs of each of happy and sad impressions using Scheffé's method of paired comparison. These data were used to construct psychological scales for the two impressions, and a statistical method was applied to test significant difference among designed impressions.

\section{Experimental Results and Discus- sion}

Figure 5 shows the obtained psychological scales with eight IEC users, and Figures 6 and 7 show the obtained eight CG lighting designs for happy and sad with arrows indicating a significant difference between the CG lightings before and after the arrow. Psychological yardsticks for a happy constructed scale are 0.19 and 0.16 for $(p<0.01)$ and $(p<$ 


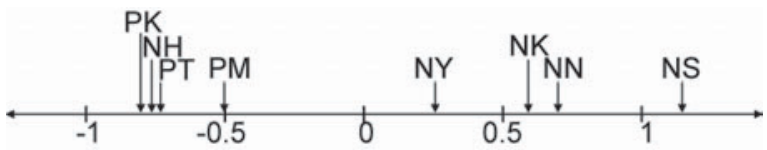

(a) happy case

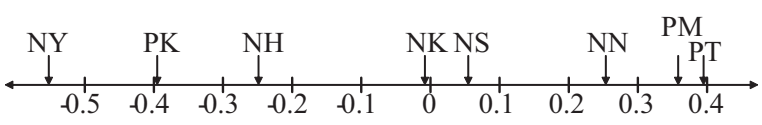

(b) sad case

Figure 5: Psychological scales constructed using Scheffé's method of paired comparison and impression levels of the eight best lightings designed by the eight subjects. See the subject marks in section 3.1.

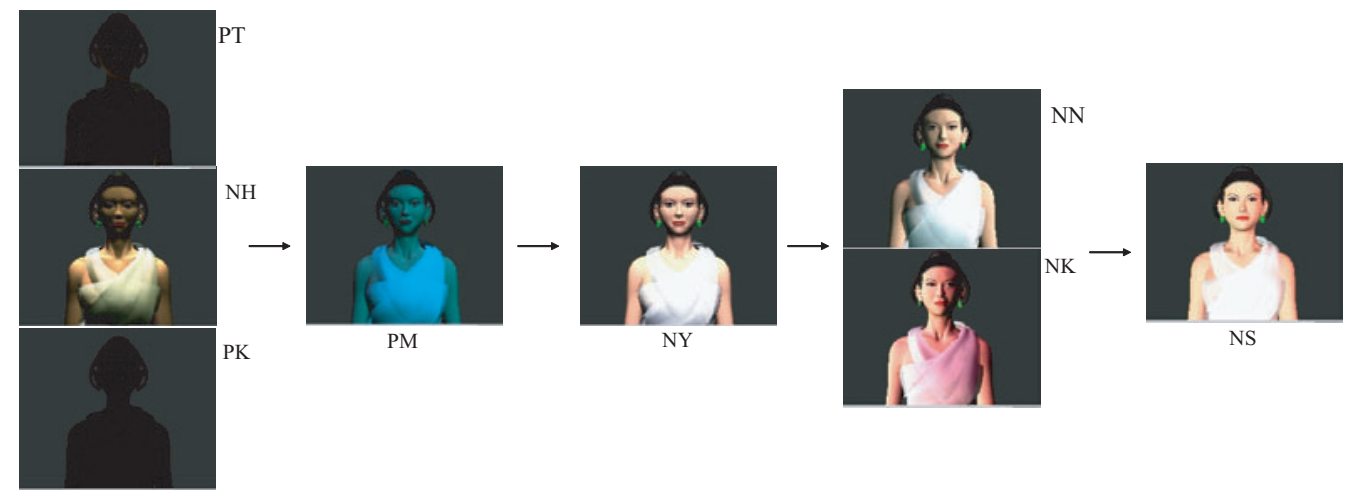

Figure 6: CG images of happy lighting designs that correspond to Figure 5(a). The arrows indicate that the CG images before and after the arrows are significantly different in happy level. See the subject marks in section 3.1.

$0.05)$, respectively, and those for a sad constructed scale are 0.24 and 0.21 for $(p<0.01)$ and $(p<0.05)$, respectively. By comparing these values on the constructed psychological scales in Figure 5 and the distances between the CG lighting designs on the scale, we can say that for the happy CG lighting, there is a significant difference between the emotional expression capability of the three schizophrenics and the five mentally healthy students with $(p<0.01)$, with the exception of subject $\mathrm{NH}$. The same conclusion cannot be made for the sad CG lighting designs, even if $(p<0.05)$.

Figure 8 shows a ranking of the IEC users based on their emotional expression range. Using the psychological scale data in Figure 5 (a) and (b), we are able to calculate the user's range of emotional expression. The range is calculated from the difference between the happy and sad scale values. Although the numerical values do not have a quantitative meaning because the two scales are constructed independently, and therefore cannot be used for interval scale measurements, the difference value can be used for an ordinal scale to generate a ranking of each user's emotional expression range.

Our experimental results show that the happy CG lighting designs made by mentally healthy subjects are significantly happier than those designed by schizophrenics and the sad expression capability looks similar, which supports our hypothesis that the dynamic expression range of happy-sad of schizophrenics is narrower than that of mentally healthy subjects.



Figure 7: CG images of the sad lighting designs that correspond to Figure 5(b). The arrows show that the CG images before and after the arrows are significantly different in sad level. See the subject marks in section 3.1.

Furtherer analysis may provide us unknown information on the symptoms or characteristics of schizophrenia. The color usage of the three patients to express a happy impression looks quite different from that of the five mentally healthy students, including subject NH whose happy design was evaluated as being less happy. Another piece of information revealed by the research was that the difficulty of this task varied considerably between the two groups; the three schizophrenics took several times longer than the students to complete the experiment, and they reported that it had been 
a tough task to evaluate the emotional impression of the images. These observations might be useful cues to understand the mental mechanism of schizophrenia and to assist diagnosis.

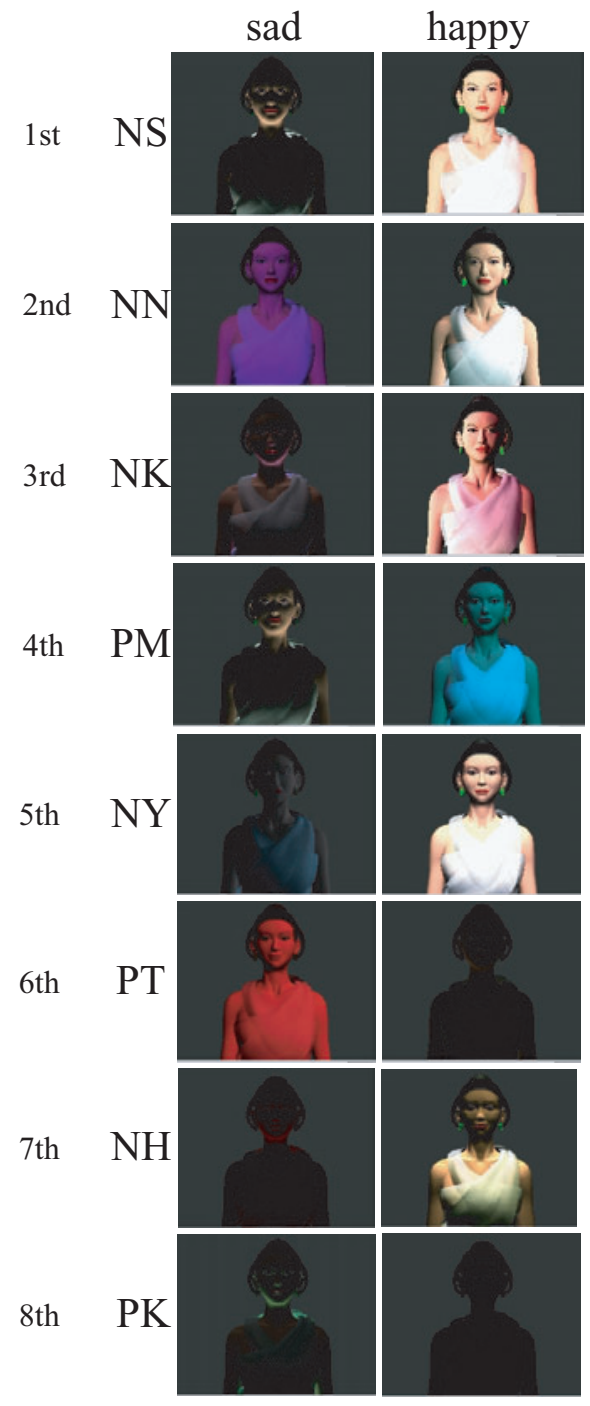

Figure 8: Rank order of happy-sad expression range obtained as a difference between two scales in Figure 5. See the subject marks in section 3.1.

\section{Conclusion}

We proposed the application of IEC to a new field, mental health measurement. We illustrated its applicability through its concrete realization in an experiment to measure the dynamic range of emotional expression capability in schizophrenics and mentally healthy subjects.

In addition to our proposal of a new direction for IEC research, our experimental results showed potential in contributing to psychiatry. If our hypothesis mentioned in sec- tion 2 is correct and it is shown that there is a relation between the emotional expression capability of schizophrenics and the level of their symptoms, history of dosing medicines, or environment, it is expected that this approach may provide new data that are helpful for psychiatric diagnostics. The application of this approach is not limited to schizophrenics, but may also prove useful for several kinds of mental diseases and in the field of therapy. We are planning to apply this technique to children who have behavioral problems as the second step of our evaluation of this approach.

It will take a long time before this approach's potential can be proven and accepted by psychiatrists or psychotherapists. However, regardless of this proof and its acceptance in psychiatry, this research has shown the application of IEC to psychiatry is a first, but big, step towards a new direction for IEC research and psychiatry.

\section{Acknowledgements}

We would like to thank 8 and 33 subjects for joining to our experiments in section 3 and thank Mr. K. Yoshimura at Ipponmatsu Hospital, Mr. K. Sato and Ms. Y. Nenoki, graduate students of Kyushu Institute of Design, for assisting these experiments. This research was supported in part by Grant-in-Aid for Scientific Research (C)(2) No.13680451.

\section{References}

[1] Aoki, K. and Takagi, H.: "3-D CG Lighting with an Interactive GA," 1st Int. Conf. on Conventional and Knowledge-based Intelligent Electronic Systems (KES'97), pp. 296-301, Adelaide, Australia (May 21-23, 1997).

[2] Aoki, K. and Takagi, H.: "Interactive GA-based Design Support System for Lighting Design in 3-D Computer Graphics," Trans. of IEICE, vol.J81-DII, no.7, pp. 16011608 (1998) (in Japanese) .

[3] Kay, S. R., Fiszbein, A, and Opler, L. A.: "The Positive and Negative Syndrome Scale for schizophrenia," Schizophr Bull, vol.13, no.2, pp. 261-276 (1987).

[4] Takagi, H.: "Interactive Evolutionary Computation: Fusion of the Capacities of EC Optimization and $\mathrm{Hu}$ man Evaluation," Proceedings of the IEEE, vol.89, no.9, pp. 1275-1296 (2001). 